\title{
ChemComm
}

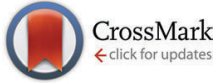

Cite this: Chem. Commun., 2016, 52,4513

Received 7th January 2016 Accepted 25th February 2016

DOI: $10.1039 / \mathrm{c} 6 \mathrm{cc} 00171 \mathrm{~h}$

www.rsc.org/chemcomm

\section{Manipulating three-dimensional gel network entanglement by thin film shearing $\dagger$}

\author{
Harshita Kumari, ${ }^{\text {a }}$ Steven R. Kline, ${ }^{\text {b }}$ Stuart R. Kennedy, ${ }^{c}$ Christopher Garvey, \\ Colin L. Raston, ${ }^{e}$ Jerry L. Atwood ${ }^{*}{ }^{\dagger}$ and Jonathan W. Steed ${ }^{* c}$
}

\begin{abstract}
Vortex fluidic mediated shearing of supramolecular gels in thin films leads to complete disruption of fluorous bis-urea derived gels. Hydrocarbon analogues however, are only partially disrupted, which emphasizes the resistance of non-fluorous bis-urea gelators towards shear. The gel structures have been studied by combining the thin film shearing with small angle neutron scattering. This technique represents a novel approach to study the effects of external stimuli on self-assembled supramolecular gel networks.
\end{abstract}

Self-assembly of supramolecular nanostructures is often a rapid and powerful process, resulting in complex structures with a number of emergent properties. However, the mechanism of formation of complex assemblies, particularly under nonequilibrium conditions, is still often speculative. Non-covalent interactions between complementary entities have led to the formation of a range of interesting materials from discrete supramolecular nanocapsules and cages to nanotubes and gel networks. ${ }^{1}$ These materials are often characterized using traditional techniques such as single-crystal X-ray diffraction (sc-XRD), PXRD, TGA, DSC and more. The structure of materials in the solution or gel phase, and the soft matter self-assembly processes that give rise to them, are less well understood due to the dynamic nature of fluid phases, where assembly depends strongly on solvation effects and dynamic solute-solvent interactions. It is of the utmost importance to know both the solid and solution phase structure to fully decode the self-assembly

\footnotetext{
${ }^{a}$ James L. Winkle College of Pharmacy, University of Cincinnati, 3225 Eden Avenue, Cincinnati, OH 45221, USA. E-mail: kumariha@ucmail.uc.edu

${ }^{b}$ NIST Center for Neutron Research, National Institute of Standards and Technology, 100 Bureau Drive, Gaithersburg, MD 20899, USA

c Department of Chemistry, Durham University, South Road, Durham DH1 3LE, UK. E-mail: jon.steed@durham.ac.uk

${ }^{d}$ Bragg Institute, Australian Nuclear Science and Technology Organization, New Illawara Road, Lucas Heights, NSW, Australia

${ }^{e}$ Flinders Centre for NanoScale Science \& Technology, School of Chemical \& Physical Sciences, Flinders University, Adelaide, SA 5001, Australia

${ }^{f}$ Department of Chemistry, University of Missouri-Columbia, 601 S. College Avenue, Columbia, MO 65211, USA. E-mail: atwoodj@missouri.edu

$\dagger$ Electronic supplementary information (ESI) available. See DOI: 10.1039/c6cc00171h
}

processes that lead to the formation of solid materials such as gels. Recently, advanced neutron scattering techniques have given considerable insight into the assembly of discrete pyrogallol[4]arene based nanocapsules and nanotubes in solution. ${ }^{2}$ We now turn these techniques to the supramolecular assembly of more complex supramolecular gel fibre networks.

The primary supramolecular structure of low molecular weight gelators (LMWG) is reasonably well understood and is thought to be based on one dimensional hydrogen bonded chains. ${ }^{3}$ However, understanding the evolution of a three-dimensional gel network from a one dimensional hydrogen bonded fibril is often speculative. The ability of a three-dimensional gel network to immobilize a continuous solvent phase present at well over $90 \%$ by mass is remarkable. Controlling the entanglement of the gel networks or manipulation of gel architecture to tune its physio-chemical properties is a challenging task. Tuning gel networks could be very useful for practical applications, such as drug release, ${ }^{4}$ sensing ${ }^{5}$ and as pharmaceutical crystallization media. ${ }^{6}$ In the present work, we have investigated the behaviour of two model gel networks using a unique combination of thin-film shearing in a recently developed vortex fluidic device (VFD) ${ }^{7}$ with small-angle neutron scattering (SANS) in order to probe the structural and mechanical properties of the gel network.

Bis-ureas are highly versatile yet relatively simple small molecule gelators capable of gelling a wide variety of polar and non-polar solvents depending on the choice of substituent groups. ${ }^{8}$ Gelators with hydrophobic substituents can gel organic solvents such as esters, ketones, alcohols, aliphatic and aromatic hydrocarbons. There have also been a few reports of polyfluorinated bis-ureas. ${ }^{9}$ Interest in fluorous compounds derives from the application of perfluorocarbons (PFCs) in wound protecting creams and for in vivo oxygen delivery. ${ }^{10}$ Fluorous-LMWGs are typically less efficient in gelating organic solvents and PFCs due to their low surface tension. Nevertheless, there are a few reported compounds that can overcome this limitation. ${ }^{11}$

Herein, we investigate the thin film shear-induced manipulation of two representative non-fluorinated and perfluoroalkyl substituted bis-urea gelators in dimethylsulfoxide (DMSO). 

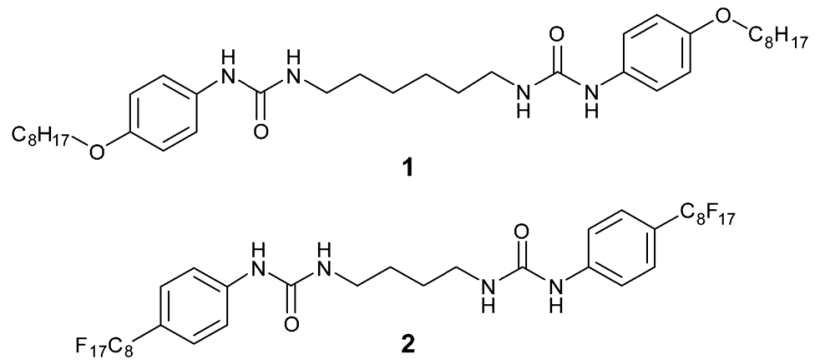

Fig. 1 Molecular structures of the fluorous and non-fluorous bis-urea gelators used in the present study.

In previous work, we have examined the thermally induced gelation and melting of gels based on these compounds using SANS and reported their rheological behaviour. ${ }^{9 b}$ These bis-urea gels exhibit intriguing physical properties. While the rigidity of gel fibres of non-fluorinated (1) and fluorinated (2) molecules are comparable (Fig. 1), the higher transparency due to fewer interchain interactions and segregation of the fluorous and hydrocarbon regions in the crystal structure of the material makes it an exciting candidate for further investigation in comparison to the more conventional hydrocarbon analogue. Interestingly, an annealing effect is observed exclusively for the fluorinated bisurea, in a way analogous to Ostwald ripening. SANS analyses showed that the structure of fluorinated bis-urea is retained upto the $T_{\text {gel }}\left(80^{\circ} \mathrm{C}\right)$ in DMSO and is fully reversible, in contrast to the structure collapsing to spherical aggregates in $N, N$-dimethylformamide (DMF) solvent. The related non-fluorinated gels, on the other hand, has thermoreversible behaviour. This intriguing difference in structural transitions was not obvious through physical observations, and was only deduced through SANS data analyses.

Apart from structural-temperature correlation, structuralconcentration effects were also studied. A change in local structure was observed for $\mathbf{2}$ as a function of concentration, but qualitative features such as fibre rigidity and cylinder radius were unaffected.

In the present study, structural correlation with respect to thin film shearing in a VFD is investigated. Because of the difficulty of preparing homogeneous gel of $\mathbf{1}$ at higher concentrations ( $5 \mathrm{wt} \%$ ), only $2 \mathrm{wt} \%$ concentration samples were subjected to thin film shearing. Compounds 1 and 2 were dissolved in $d_{6}$-DMSO at $2 \mathrm{wt} \%$ each and stirred gently with a glass rod to obtain uniform gels in a borosilicate $20 \mathrm{~mm}$ NMR tube. The tube was then placed in hot water solution to allow for the formation of a clear uniform solution prior to gelation. On cooling down, the tube was placed in the VFD microfluidic platform and rotated (Fig. 2). Deuterated solvent, $d_{6}$-DMSO, was used to improve the contrast and to enhance coherent scattering for subsequent small-angle neutron scattering measurements. Note that the $20 \mathrm{~mm}$ NMR tube chosen for sample preparation also acts as a sample holder for the VFD. ${ }^{7,12}$

Processing using the VFD involves rapidly rotating the glass sample tube between two bearings, inclined at an angle $\theta$ relative to the horizontal position. The speed can be controlled within $1 \mathrm{rpm}$,

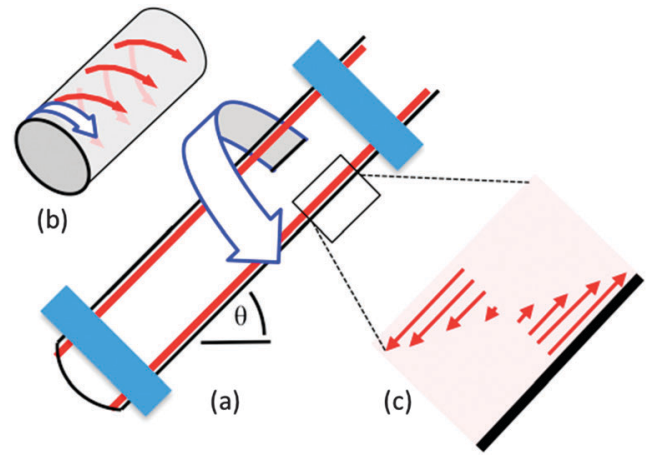

Fig. 2 (a) The Vortex Fluidic Device (VFD) ${ }^{7}$ showing the tilt angle $\theta$, with (b) showing the direction of liquid flow as red arrows, and (c) highlighting the Stewartson/Ekman layers in the dynamic thin film.

for speeds high enough to ensure that a vortex is maintained to the hemispherical base of the tube, yet without any of liquid exiting the top of the tube. This is important in maintaining a more uniform thin film and associated shearing, and represents the so called confined mode of operation of the VFD. ${ }^{7}$ The VFD imparts mechanoenergy within the thin film, with the fluid forming Stewartson/Ekman layers on the sidewall of tube which arise from the acceleration of liquid against gravity. The optimal performance of the VFD is obtained for rotating speed ranging from $2000 \mathrm{rpm}$ to $9000 \mathrm{rpm}$, for tilt angles, $\theta>0^{\circ}$. In the present work a rotation speed of $5000 \mathrm{rpm}$ and a tilt angle of $45^{\circ}$ were used. This tilt angle corresponds to the maximum cross vector of centrifugal force and gravity, and is optimal for a number of applications of the VFD, including exfoliating graphene from graphite, ${ }^{13}$ bending carbon nanotubes, ${ }^{14}$ protein folding, ${ }^{15}$ and chemical reactivity and selectivity. ${ }^{7}$ The gel samples were sheared in this confined mode for 5 minutes. The continuous flow mode of operation of the $\mathrm{VFD}^{7}$ was not applicable for throughput of gels, and in this context the confined mode of operation of the VFD is well suited for probing the structure of self organised systems in general.

The resultant VFD-treated samples of $\mathbf{1}$ and $\mathbf{2}$ were then studied by SANS after a time interval of 1 hour on the QUOKKA Instrument at the Bragg Institute, Australian Nuclear Science and Technology Organisation (ANSTO), Sydney, NSW. SANS is a reciprocal space method, similar to XRD, which provides threedimensional real space information of the sample constructed by modelling their scattering curves. The resultant fit is a true statistical perspective of the structure under investigation.

A total of $0.6 \mathrm{~mL}$ of the confined mode VFD-treated samples ( $5000 \mathrm{rpm}, 5$ minutes, $\theta=45^{\circ}$ ) was transferred by pipette to a $1 \mathrm{~mm}$ path length demountable titanium SANS cell that is mounted onto the SANS sample holder. Samples were in an intermediate sol-gel state and behaved as a viscous liquid and hence were easily transferred from the glass (NMR) tube to the SANS sample holder. SANS measurements were conducted at $25{ }^{\circ} \mathrm{C}$. Neutrons of wavelength $\lambda=5 \AA$ with a full width halfmaximum of $\Delta \lambda / \lambda=12 \%$ were used. The sample to detector distances of $1.3 \mathrm{~m}, 4.5 \mathrm{~m}$ and $13 \mathrm{~m}$ were used to cover the $q$ range of $0.013 \AA^{-1}<q<0.534 \AA^{-1}$, where $q=(4 \pi / \lambda) \sin (\theta / 2)$ ( $q=$ scattering vector; $\theta=$ scattering angle). The scattered data 
for the samples were corrected for the background, empty cell scattering and the sensitivity of the individual detector pixels. The corrected data were then placed on an absolute scale and structures were modelled using Igor Pro software, ${ }^{16}$ (provided by NIST) using a $q$-resolution function and smearing effect for structure solution.

SANS scattering curves for native fluorinated and fluorinated bis-urea gelators (no VFD processing as control experiments) fit well to a spherical fractal and fractal flexible cylinder models. ${ }^{9 b}$ The word 'fractal' is akin to a 'crystallographic lattice' and refers to an infinite complex pattern of repeating units that exhibits at every scale/unit cell. A gel network can be considered as a complex non-crystallographic lattice that consists of fractals or fibers of defined length scale that exists in defined periodicity. Comparing statistics of models suggests that both fractal and fractal flexible cylinder model fits well to the scattering data of $\mathbf{1}$ and $\mathbf{2}$. The correlation length for sample $\mathbf{1}$ (non-fluorinated) is an order of magnitude higher than that of sample 2 (fluorinated). However, the ratio between contour lengths and Kuhn lengths is about the same, indicating that the fibers in both cases are rigid. The Gaussian fitting at the high $q$ region yields the peak height ratios of 2 which suggest that both fluorinated and nonfluorinated gels possess a lamellar structure with d spacing of $\approx 39 \AA$ and $\approx 35 \AA$, respectively. ${ }^{9 b}$

Given the similarity in rigidity and lamellar structures, we expected both materials to behave similarly under thin film shear. In case of 2, shearing in the VFD under confined mode yielded a flat scattering intensity (Fig. 3). An overlay of the native and VFD treated sample shows a complete disruption of the gel structure for both low and high $q$ length scales. This suggests that the shear within the thin film at $5000 \mathrm{rpm}$ was sufficient to completely break the gel network. Interestingly, the resultant scattering curve shows no features, indicating the absence of any discrete architecture post thin-film shearing.

Confined mode thin film shearing of the non-fluorinated gel in DMSO, on the other hand, shows that the local stacking of bis-urea molecules in the gel is preserved. The high $q$ region refers to local structure at shorter distances. The Gaussian fitting of high $q$ data for 1 yields $1: 2$ ratio of peak positions suggesting the presence of lamellar structures (Fig. 4). In addition, high $q$ Gaussian fitting of 1 gives a $d$-spacing of $\approx 35 \AA$ for

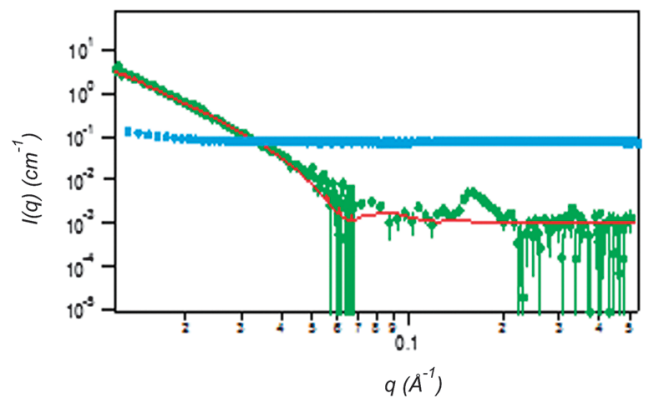

Fig. 3 Overlay of scattering curves of native and VFD treated fluorinated bis-urea gel (2) showing disruption of gel upon VFD treatment. Green dots: SANS curve of 2. Blue dots: SANS curve 2 treated with VFD. Red curve: fitting to a smeared fractal cylinder model.

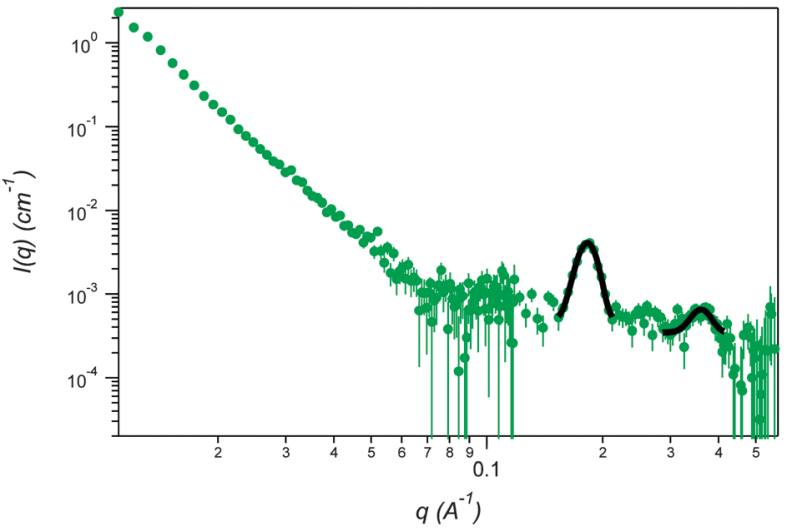

Fig. 4 Gaussian fit of high $q$ data of VFD treated non-fluorinated bis-urea (1) gelator showing fittings of two peak positions (black curve). Green dots: scattering points of VFD treated sample 1; black curve: Gaussian fit.

both original and thin-film sheared gels, which correlates with the opacity of non-fluorinated gels.

An overlay of scattering curves of native and VFD treated non-fluorinated bis-urea gels shows a similar scattering profile (Fig. 5).

The low $q$ data fitting of native gel 1 to a fractal flexible cylinder model yields a correlation length of $1650 \AA$, fractal dimension of 3 , contour length of $\approx 37 \AA$, Kuhn length of $\approx 40 \AA$ and cylinder radius of $\approx 54 \AA$. The primary difference in the low $q$ data fitting of VFD treated gel 1 is the value of contour length $\approx 10 \AA$ and Kuhn length $\approx 10 \AA$; other parameters are similar (within error) to those of the as-prepared gel network. Contour length represents the end-to-end length of the polymer chain if it were fully stretched out whereas Kuhn length is the average length over which the flexible cylinder blocks can be considered rigid. No difference between contour and Kuhn length of VFD treated gels suggests that the gel fibers are still rigid; however, the reduction in Contour and Kuhn lengths suggests that the gel's building blocks are broken down into smaller pieces. Note that the high $q$ Gaussian fitting reveals a lamellar structure whereas the low $q$ fitting shows breaking of the gel into smaller units. It indicates that the pre-VFD treated sample is made of large building blocks that are possibly stacks

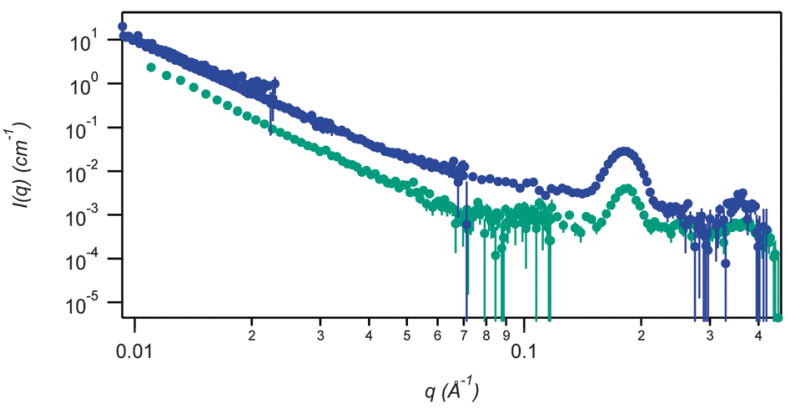

Fig. 5 Overlay of scattering curves of native and VFD treated non-fluorinated bis-urea gel (1) showing no disruption of the gel structure upon thin-film shearing. Green dots: VFD treated non-fluorinated bis-urea in DMSO; Blue dots: non-fluorinated bis-urea gel in DMSO. 
of flat aggregates of the molecules. VFD processing breaks these stacks into smaller units, but the gel is strong enough that the local lamellar structure and the overall gel persists.

Both bis-urea gels are based on urea $\alpha$-tape-type hydrogen bonding interactions supported in the case of $\mathbf{1}$ by van der Waals interactions between aliphatic residues. The stacking interactions here appear to tolerate the shearing better than the stacking interactions between the fluorinated regions in 2 , in which thin film shearing was sufficient to break both local and bulk structure of the fluorinated bis-urea gel.

Mechanical shearing with a conventional parallel plate geometry rheometer of 1 and 2 yields $G^{\prime}$ value of $1.32 \times 10^{3} \mathrm{~Pa}$ and $1.51 \times 10^{4} \mathrm{~Pa}$, respectively, suggesting a higher elastic modulus for the fluorinated gelator. ${ }^{9 b}$ In addition, 2 has an yield stress value of $203 \mathrm{~Pa}$ at $1 \mathrm{wt} \%$ whereas 1 has an yield stress value of $3 \mathrm{~Pa}$ at $3 \mathrm{wt} \%$, indicating that more stress is required to deform fluorinated gelator plastically, consistent with its higher elastic modulus. ${ }^{9 b}$ Thin-film shearing with VFD, on the other hand, shows complete breakage of fluorinated gelator and partial breakage of non-fluorinated gelator at $5000 \mathrm{rpm}$. SANS provides a more qualitative insight into thin-film shearing.

Combined VFD and SANS analyses has shown that gel networks are sensitive to high shear rate and that shearing can affect local structure and higher order aggregation. The SANS analyses has revealed that although the strength/rigidity of fibers is similar for two chemically different gels (fluorinated versus non-fluorinated), ${ }^{9 b}$ the gel network may be far less resistant to mechanical thin film shear and recover less well if fewer intermolecular interactions are supporting the primary hydrogen bonded assembly. Also, the selfassembly process of gel molecules involves a local network of molecules (high $q$ ) which corresponds primarily to short stacks of parent molecules and a bulk network/mesh (low q) which corresponds to the lateral interlinking of fibers between self-assembled bis-urea molecules. Thin film shearing in the VFD can be used to manipulate and tune the local or bulk structure.

In conclusion, this is the first study on probing the structure of gels under thin-film shear. This involved a novel combination of thin film processing using the confined mode of operation of the VFD, with static SANS. Cohesive thin film shearing and simultaneous structural analyses established different response to a fluorinated bis-urea gel versus a non-fluorinated bis-urea gel network. This technique has the potential to provide insight into the structural basis for emergent materials phenomena such as thixotropy. ${ }^{17}$ Future work will focus on real-time SANS with VFD calibration studies that will allow visualization of structures during the course of shearing.

We thank National Science Foundation and UK Engineering and Physical Sciences Research Council (grant reference EP/J013021/1) and the Australian research Council and the Government of South Australia for funding. The underlying research data for this paper is available in accordance with EPSRC open data policy from http://dx.doi.org/10.15128/kh04dp681.

\section{Notes and references}

1 (a) S. J. Dalgarno, N. P. Power and J. L. Atwood, Coord. Chem. Rev., 2008, 252, 825-841; (b) P. Jin, S. J. Dalgarno and J. L. Atwood, Coord. Chem. Rev., 2010, 254, 1760-1768; (c) G. W. Orr, L. J. Barbour and J. L. Atwood, Science, 1999, 285, 1049-1052; (d) L. Avram and Y. Cohen, Org. Lett., 2003, 5, 3329-3332; (e) P. Ballester and G. Gil-Ramirez, Proc. Natl. Acad. Sci. U. S. A., 2009, 106, 10455-10459, S10455/10451-S10455/ 10410; $(f)$ M. M. Conn and J. Rebek, Chem. Rev., 1997, 97, 1647-1668; (g) F. Corbellini, R. M. A. Knegtel, P. D. J. Grootenhuis, M. CregoCalama and D. N. Reinhoudt, Chem. - Eur. J., 2005, 11, 298-307; (h) T. Douglas and M. Young, Nature, 1998, 393, 152-155; (i) C. J. Kuehl, Y. K. Kryschenko, U. Radhakrishnan, S. R. Seidel, S. D. Huang and P. J. Stang, Proc. Natl. Acad. Sci. U. S. A., 2002, 99, 4932-4936; (j) Y. Kuang, Y. Gao and B. Xu, Chem. Commun., 2011, 47, 12625; (k) S. Sajeesh, K. Bouchemal, V. Marsaud, C. Vauthier and C. P. Sharma, J. Controlled Release, 2010, 147, 377-384.

2 H. Kumari, C. A. Deakyne and J. L. Atwood, Acc. Chem. Res., 2014, 47, 3080-3088.

3 (a) R. G. Weiss, J. Am. Chem. Soc., 2014, 136, 7519-7530; (b) G. Yu, X. Yan, C. Han and F. Huang, Chem. Soc. Rev., 2013, 42, 6697; (c) A. Y.-Y. Tam and V. W.-W. Yam, Chem. Soc. Rev., 2013, 42, 1540; (d) J. Zhang, H.-F. Chow, M.-C. Chan, G. K.-W. Chow and D. Kuck, Chem. - Eur. J., 2013, 19, 15019-15025.

4 (a) J. R. Moon, Y. S. Jeon, D. J. Chung, D. Kim and J.-H. Kim, Macromol. Res., 2011, 19, 515-518; (b) V. Jokanović, B. Čolović, M. Dutour Sikirić and V. Trajković, Ultrason. Sonochem., 2013, 20, 535-545.

5 C. Zhou, J. Gao, L. Zhang and J. Zhou, Anal. Chim. Acta, 2014, 812, 129-137.

6 (a) J. Buendía, E. Matesanz, D. K. Smith and L. Sánchez, CrystEngComm, 2015, 17, 8146-8152; (b) A. Dawn, K. S. Andrew, D. S. Yufit, Y. Hong, J. P. Reddy, C. D. Jones, J. A. Aguilar and J. W. Steed, Cryst. Growth Des., $2015,15,4591-4599$.

7 L. Yasmin, X. Chen, K. A. Stubbs and C. L. Raston, Sci. Rep., 2013, 3, 2282.

8 (a) Y. Li and M. Liu, Chem. Commun., 2008, 5571, DOI: 10.1039/ b812567h; (b) J. van Esch, R. M. Kellogg and B. L. Feringa, Tetrahedron Lett., 1997, 38, 281-284; (c) F. S. Schoonbeek, J. H. van Esch, R. Hulst, R. M. Kellogg and B. L. Feringa, Chem. - Eur. J., 2000, 6, 2633-2643; (d) M. Yamanaka and H. Fujii, J. Org. Chem., 2009, 74, 5390-5394.

9 (a) J. Gladysz and M. Jurisch, in Fluorous Chemistry, ed. I. T. Horvath, Springer, Berlin, Heidelberg, 2012, ch. 282, vol. 308, pp. 1-23; (b) H. Kumari, S. E. Armitage, S. R. Kline, K. K. Damodaran, S. R. Kennedy, J. L. Atwood and J. W. Steed, Soft Matter, 2015, 11, 8471-8478.

10 J. G. Riess, Tetrahedron, 2002, 58, 4113-4131.

11 (a) P. Terech, V. Rodriguez, J. D. Barnes and G. B. McKenna, Langmuir, 1994, 10, 3406-3418; (b) E. Krieg, H. Weissman, E. Shimoni, A. Bar On and B. Rybtchinski, J. Am. Chem. Soc., 2014, 136, 9443-9452; (c) M. George, S. L. Snyder, P. Terech, C. J. Glinka and R. G. Weiss, J. Am. Chem. Soc., 2003, 125, 10275-10283; (d) J. Loiseau, M. Lescanne, A. Colin, F. Fages, J.-B. Verlhac and J.-M. Vincent, Tetrahedron, 2002, 58, 4049-4052; (e) J. Gan, M. El Bakkari, C. Belin, C. Margottin, P. Godard, J.-L. Pozzo and J.-M. Vincent, Chem. Commun., 2009, 5133, DOI: 10.1039/b908820b.

12 J. Britton, S. B. Dalziel and C. L. Raston, $R S C$ Adv., 2015, 5, 1655-1660. 13 X. Chen, J. F. Dobson and C. L. Raston, Chem. Commun., 2012, 48, 3703. 14 K. Vimalanathan, X. Chen and C. L. Raston, Chem. Commun., 2014, 50, 11295.

15 T. Z. Yuan, C. F. G. Ormonde, S. T. Kudlacek, S. Kunche, J. N. Smith, W. A. Brown, K. M. Pugliese, T. J. Olsen, M. Iftikhar, C. L. Raston and G. A. Weiss, ChemBioChem, 2015, 16, 393-396.

16 S. R. Kline, J. Appl. Crystallogr., 2006, 39, 895-900.

17 (a) X. Yu, X. Cao, L. Chen, H. Lan, B. Liu and T. Yi, Soft Matter, 2012, 8, 3329; (b) K. Liu and J. W. Steed, Soft Matter, 2013, 9, 11699; (c) W. Weng, A. M. Jamieson and S. J. Rowan, Tetrahedron, 2007, 63, 7419-7431. 